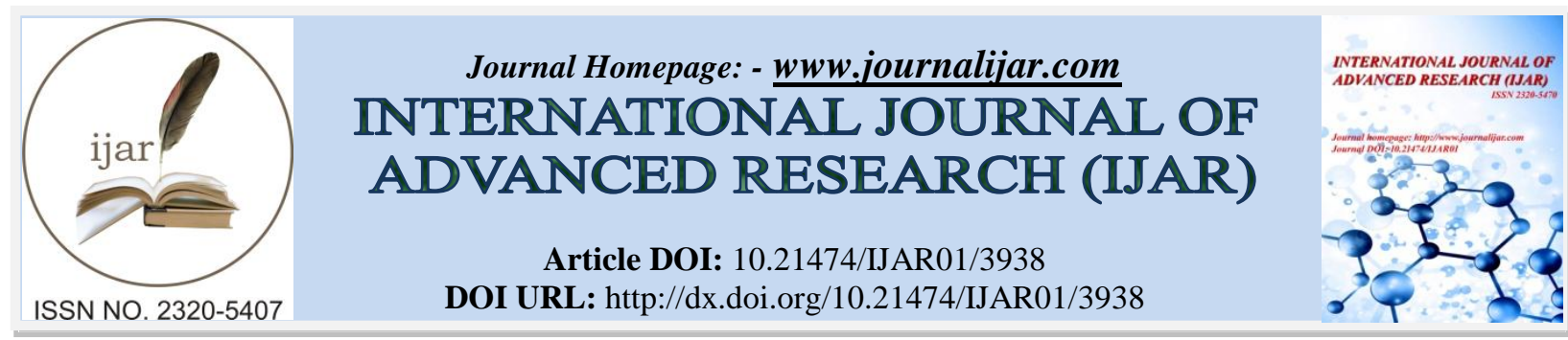

RESEARCH ARTICLE

\title{
SIGNIFICANCE OF VITAMIN -D AND INSULIN RESISTANC IN POLYCYSTIC OVARIAN SYNDROME IN MANIFESTING RHEUMATOID ARTHRITIS.
}

T.Uma ${ }^{*}$, D.S.S.Girija Vani ${ }^{2}$ and R. Vijayaraghavan ${ }^{3}$.

1. Department Of Biochemistry Ph.D scholar Saveetha University, Chennai, Tamilnadu, India.

2. Department Of Biochemistry, Saveetha University, Chennai, Tamilnadu, India..

3. Director Of Research, Saveetha University, Chennai, Tamilnadu, India.

\section{Manuscript Info}

\section{Manuscript History}

Received: 12 February 2017

Final Accepted: 15 March 2017

Published: April 2017

Key words:-

Insulin resistance, Oxidative stress, PCOS

\section{Abstract}

Aim\& objectives: Polycystic ovary syndrome (PCOS) is the most common female endocrine disorder; It is a heterogeneous androgen excess disorder with different degrees of reproductive and metabolic dysfunctions. The objective of this study is to determine whether serum vitamin $\mathrm{D}$ and insulin resistance manifest to Rheumatoid Arthritis in women with PCOS are not.

Materials and methods:- The study includes total of 80 Subjects within the age group of 20 to 50years were selected and divided into two groups. Group I consisted of 50 diagnosed female patients of PCOD. $5 \mathrm{ml}$ of blood sample was obtained from the participant under all aseptic precautions after 12 hours of fasting. $2 \mathrm{ml}$ is transferd to EDTA and ramaing blood in plain tubes allowed to clot at 1 hour and centrifuged at $2000 \mathrm{rpm}$ for $10 \mathrm{mits}$ to obtain serum. The Serum samples are collected for estimation of is glucose ,Insulin , vitamin D, insulin resistance index (HOMA - IR), and anti -ccp are analysed

Results: Women with PCOS had significantly higher FBS(112 \pm 15.9$)$ $(\mathrm{P}<0.01)$ than the control subjects $(83.30 \pm 11.95)$, Women with PCOS had significantly higher insulin values $(23 \pm .9) \quad(\mathrm{P}<0.01)$ than the control subjects $(83.30 \pm 11.95)$, Women with PCOS had significantly higher HOMA-IR $(112 \pm 15.9) \quad(\mathrm{P}<0.01)$ than the control subjects(83.30 \pm 11.95$)$, Women with PCOS had significantly higher VITAMIN $-\mathrm{D}(112 \pm 15.9) \quad(\mathrm{P}<0.01) \quad$ than the control subjects(83.30 \pm 11.95$)$.

Conclusion: Vitamin D is not only a regulator of bone and mineral metabolism, but also a potent immunomodulator linked to many major human diseases including glucose homeostasis and insulin resistance. Insulin resistance encourages oxidative stress because hyperglycemia and higher levels of free fatty acids lead to ROS production. Thus, insulin may defend against pro-inflammatory responses to hyperglycemia by acting as an anti-inflammatory agent. Proinflammatory cytokines seem to have beneficial effects on the insulin resistance observed in patients with RA and hormonal mechanisms involved in RA.

Copy Right, IJAR, 2017,. All rights reserved. 


\section{Introduction:-}

Polycystic ovary syndrome (PCOS) is the most common female endocrine disorder; It is a heterogeneous androgen excess disorder with different degrees of reproductive and metabolic dysfunctions. Metabolic disturbances including insulin resistance, hyperinsulinemia and dyslipidemia, are common features in the majority of women with PCOS . Women with PCOS may also be at elevated risk of vitamin D deficiency (VDD). In contrast to a prevalence of $20 \%-48 \%$ among the general adult population, a relative higher prevalence of VDD is observed among women with PCOS Additionally, positive associations of VDD with some well-known co morbidities of PCOS including type 2 diabetes, insulin resistance, metabolic syndrome, and cardiovascular diseases, are reported . In this regard, an increasing number of studies have been conducted to investigate the specific relationship between vitamin $\mathrm{D}$ status and PCOS. (1)

Vitamin D may play a role in glucose metabolism by enhancing insulin synthesis and release, and increasing insulin receptor expression or suppression of proinflammatory cytokines that possibly contribute to the development of insulin resistance [2]. The effect of vitamin D on metabolic and reproductive dysfunctions in PCOS may be mediated by insulin resistance. Reproductively, insulin resistance increases hyperandrogenism through insulin increasing ovarian androgen production, and reducing sex hormone-binding globulin (SHBG) production [3]. Metabolically, insulin resistance is associated with an increased risk for impaired glucose tolerance, type 2 diabetes mellitus and cardiovascular disease [4]. Therefore, vitamin D may play a key role in the development of PCOS.

Insulin resistance is a common feature of PCOS affecting 50-70\% of women with the disorder. Polycystic ovary syndrome (PCOS) is an exceptionally common disorder of premenopausal women characterized by hyperandrogenism and chronic anovulation. Its etiology remains unknown. A biologic response to insulin that is less than normal is defined as insulin resistance. A relationship between insulin and hyperandrogenism has been observed from several different vantage points. hyperinsulinemia play important roles in the pathogenesis of PCOS (Dunaif A, 1997). (5)IR and hyperinsulinemia appears to interfere with ovarian steriodogenetic defects as well as anovalatory mechanism (Poretsky L P-999). These two factors produce the hyperandrogenism interfering with pituitary ovarian axis, leading to increased LH levels, anovulation, amenorrhea and infertility. PCOS is associated with an increased risk of metabolic complications including impaired glucose tolerance, type 2 diabetes, hypertension, dyslipidemia. Several studies have documented that atleast 30-40\% of PCOS patients were obese or over weight and obesity has an aggravating role in PCOS. (Diamantri - Kandarakis et al 1999, Azziz et al 2004)

\section{Materials and Methods:-}

The study was approved by the institutional Human Ethical Committee (IHEC) at saveetha university . Informed consent was obtained from participants. The objectives of the study were explained and a written concept was taken from each subject. The data on family history and personal history were collected through standard questionnaire.

Permission was also taken from hospital for sample \& data collection and analysis. $5 \mathrm{ml}$ of blood sample was obtained from the participant under all aseptic precautions.The study was carried out at Rajiv Gandhi Institute Of Medical Sciences \& general hospital, Srikakulam, Andhra Pradesh in collaboration with Department of Obstetrics and Gynecology. The study includes the patients diagnosed as PCOD by using revised diagnostic criteria (Rotterdam criteria) two of the three criteria is required to be present Oligomenorrhea/amenorrhea, Polycystic ovaries on ultrasound, Clinical or biochemical signs of hyperandrogenism. Diagnosed cases of diabetes mellitus, thyroid dysfunction, Cushing's syndrome, congenital andrenal hyperplasia, hyperproloctinemia, androgen secreting tumor.Subjects taking medicines like ovulation induction agents, hormonal drugs and current or previous use of oral contraceptives within last 6 months are excluded from the study. Based on inclusion and exclusion criteria, a total of 80 Subjects within the age group of 20 to 50years were selected and divided into two groups. Group I consisted of 50 diagnosed female patients of PCOD. Group II comprised of 30 control group with normal menstrual cycles. ).

$5 \mathrm{ml}$ of blood sample was obtained from the participant under all aseptic precautions after 12 hours of fasting . $2 \mathrm{ml}$ is transferd to EDTA and ramaing blood in plain tubes allowed to clot at 1 hour and centrifuged at 2000rpm for 10 mits to obtain serum. The Serum samples for estimation of is glucose by Glucose oxidase and peroxidase method which is analysed calorimetrically. Insulin, vitamin D and anti $-\mathrm{ccp}$ are analysis is carried out by chemiluminescence immunoassay by using Access 2, Beckman Coulter Manufactured by: Beckman Coulter, Inc.4300 N. Harbor Blvd.Fullerton, CA 92835 U.S.A. 
Insulin resistance will be measured by homeostasis model assessment insulin resistance index (HOMA - IR). HOMA-IR = Fasting serum insulin $(\mathrm{mU} / \mathrm{ml}) \times$ fasting plasma glucose $(\mathrm{mmol} / \mathrm{l})$

\section{5}

\section{Statistical Analysis:-}

Data was expressed as mean \pm S.D. For comparison of all quantitative variables between control and PCOS groups, the unpaired t-test was used and association between the parameters were assayed by Pearson correlation analysis. Statistical significance was accepted at $\mathrm{P}<0.05$. All statistical analysis were performed using SPSS Program .

\begin{tabular}{|l|l|l|l|l|l|l|l|l|}
\hline \multirow{2}{*}{ Sl.no } & \multirow{2}{*}{ Variable } & \multicolumn{2}{l|}{ controls } & \multicolumn{2}{l|}{ PCOS } & p-value SIGNIFICANCE \\
\cline { 3 - 8 } & & $\mathrm{N}$ & Mean & S.D & N & Mean & S.D & \\
\hline 1 & FBS & 30 & 83.30 & 11.95 & 50 & 112.30 & 15.89 & $<0.01$ HS \\
\hline 2 & Insulin & 30 & 15.50 & 2.87 & 50 & 23.38 & 5.39 & $<0.01 \mathrm{HS}$ \\
\hline 3 & HOMA-IR & 30 & 3.20 & 0.82 & 50 & 6.62 & 2.15 & $<0.01 \mathrm{HS}$ \\
\hline 4 & Vitamin-D & 30 & 59.63 & 12.41 & 50 & 31.32 & 5.24 & $<0.01 \mathrm{HS}$ \\
\hline
\end{tabular}

\section{Results:-}

Women with PCOS had significantly higher FBS $(112 \pm 15.9)(\mathrm{P}<0.01)$ than the control subjects(83.30 \pm 11.95$)$, Women with PCOS had significantly higher insulin values $(23 \pm .9)(\mathrm{P}<0.01)$ than the control subjects $(83.30 \pm 11.95)$, Women with PCOS had significantly higher HOMA-IR $(112 \pm 15.9)(\mathrm{P}<0.01)$ than the control subjects $(83.30 \pm 11.95)$, Women with PCOS had significantly higher VITAMIN $-\mathrm{D}(112 \pm 15.9) \quad(\mathrm{P}<0.01)$ than the control subjects(83.30 \pm 11.95$)$,

\begin{tabular}{|l|l|l|l|}
\hline \multirow{2}{*}{ Group } & Anti CCP & \multirow{2}{*}{ Total } \\
\cline { 2 - 3 } & $\langle\mathbf{1 7}$ & $\mathbf{> 1 7}$ & \\
\hline \multirow{2}{*}{ Control } & 30 & 0 & 30 \\
\cline { 2 - 4 } & $100 \%$ & $0 \%$ & $100 \%$ \\
\hline \multirow{2}{*}{ PCOD } & 44 & 6 & 50 \\
\cline { 2 - 4 } & $88 \%$ & $12 \%$ & $100 \%$ \\
\hline Fisher exact test; $\mathbf{p}=\mathbf{0 . 0 5} ; \mathbf{S}$ &
\end{tabular}

Fig 1:- Comparison of patients with Controls - FBS-Glucose (Mean)

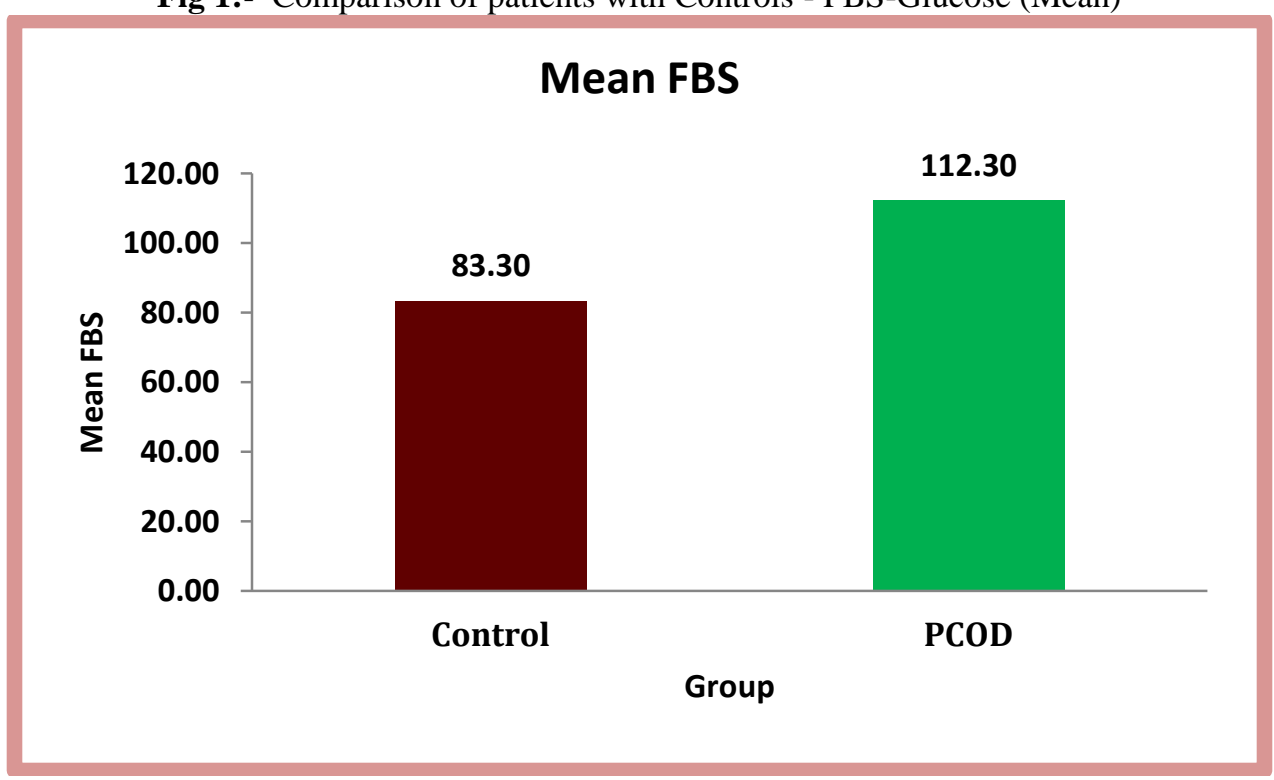


Fig 2:- Comparison of patients with Controls - Insulin (Mean)

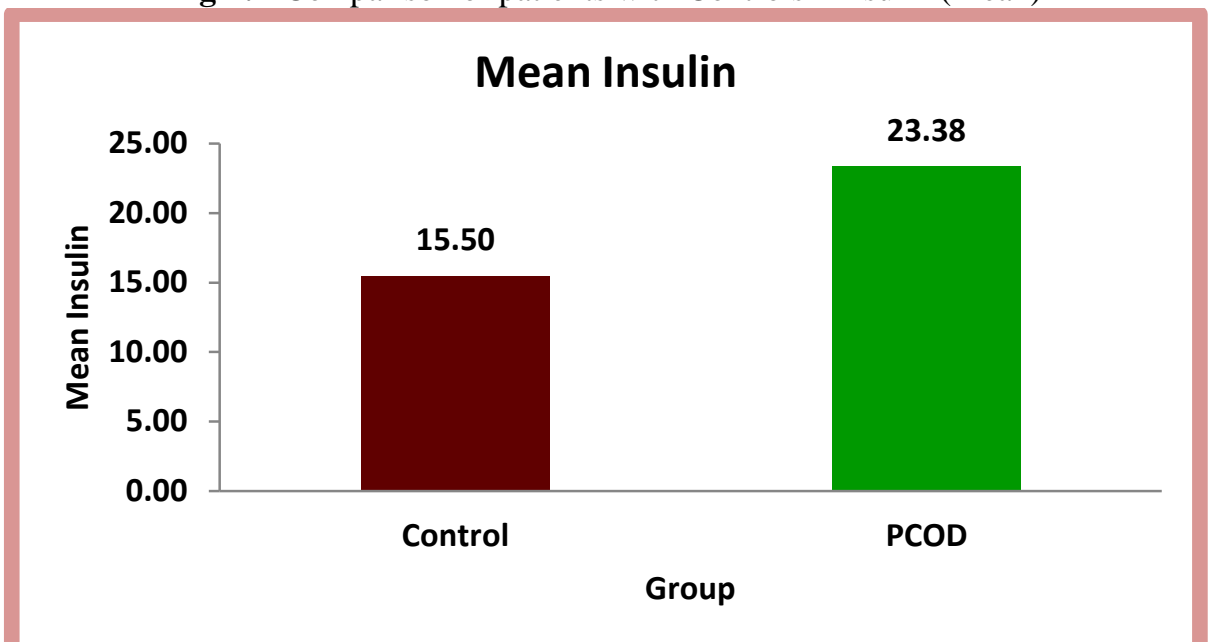

Fig 3:- Comparison of patients with Controls - HOMA IR (Mean)

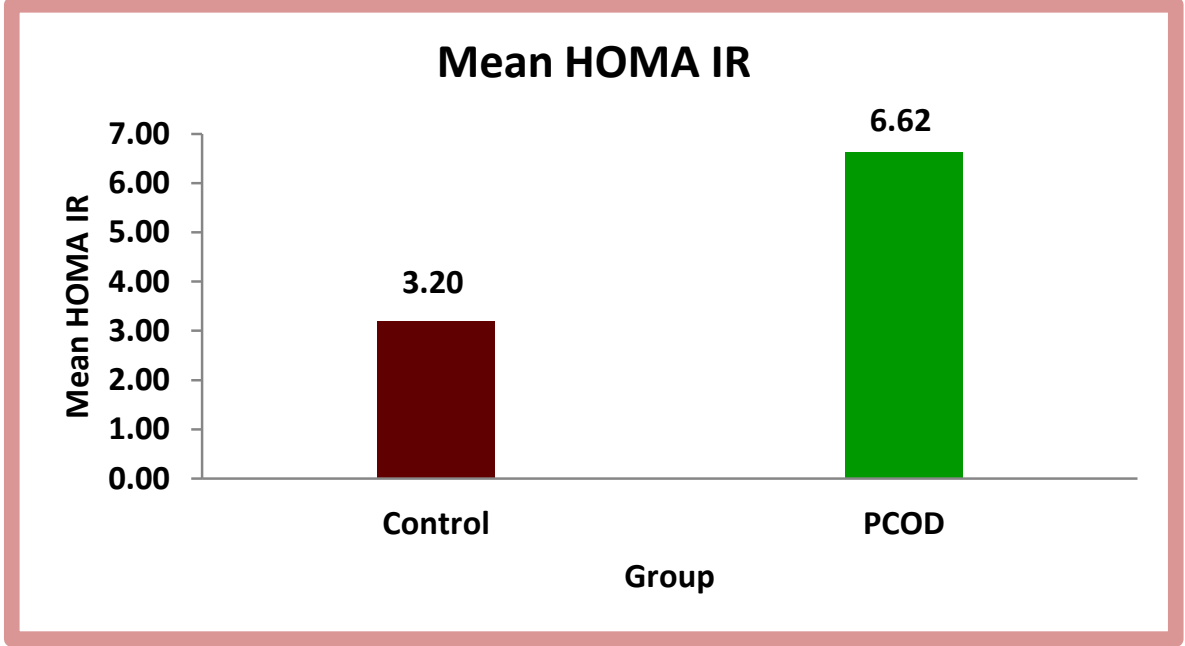

Fig 4:- Comparison of patients with Controls - vitamin-D (Mean)

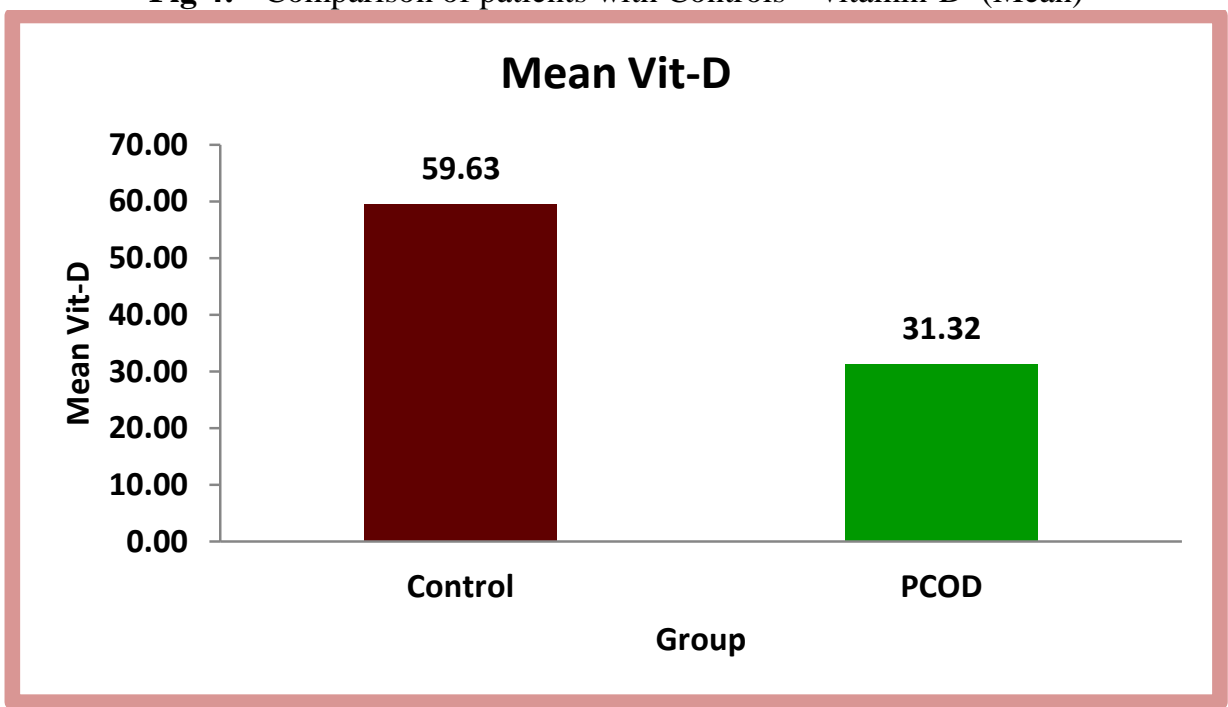


Fig 5:- Comparison of patients with Controls - Anti ccp

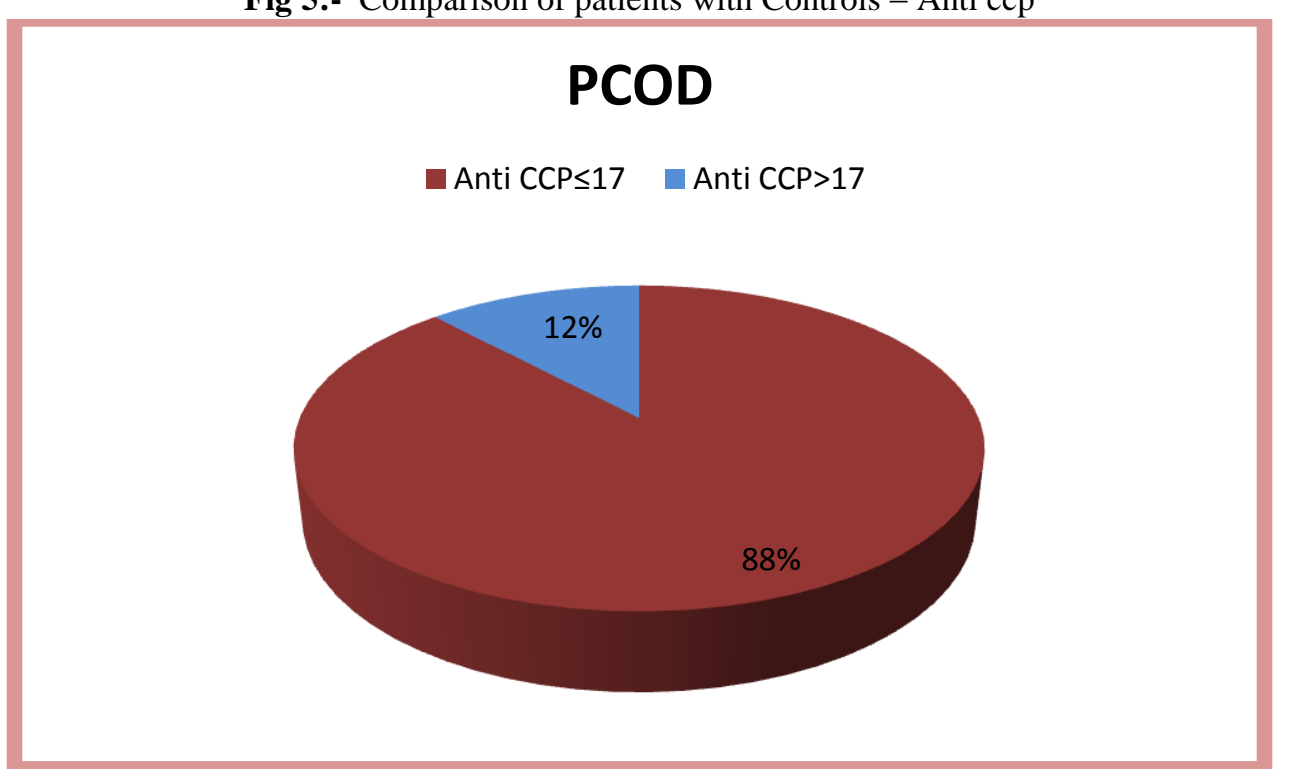

\section{Disscussion:-}

The present study supports that serum vitamin D status is decreased in pcos which is related to metabolic and hormonal dysfunctions in women with PCOS. Low $25(\mathrm{OH}) \mathrm{D}$ levels are found to be significantly correlated with

insulin resistance in women with PCOS Among women with PCOS, those with vitamin D deficiency were more likely to have measures of dysglycemia compared to those without vitamin D deficiency. Similarly, lower vitamin D levels were positively correlated with markers of dysglycemia, support a significant difference in serum vitamin D levels between women with and without PCOS.(1)

Vitamin D deficiency (VDD) is common in women with and polycystic ovary syndrome (PCOS) and may be associated with metabolic and endocrine disorders in PCOS. There is much evidence, that in addition to sex steroid hormones, the classic regulators of reproduction, Vitamin also modulates Reproductive processes in women; its nuclear receptor has been identified in the uterus, oviduct, ovary, placenta, and fetal membranes. A recent observational study by Velija-Asimi et al. found that 68\% (41 out of 60) PCOS patients had VDD, of which $54 \%(n=22)$ were obese and $46 \%$ were non-obese $(n=19)$ [6]. Another study also reported a higher prevalence of VDD in obese women with PCOS than in lean women with PCOS (70\% vs. 60\%) [7].

There is some evidence suggesting that vitamin D deficiency may be involved in the pathogenesis of insulin resistance and metabolic syndrome in PCOS The effects of vitamin D are mediated via both genetic and cellular pathways. Vitamin D regulates gene transcription through nuclear vitamin D receptors (VDR) that are distributed across various tissues, including skeleton, parathyroid glands and the ovaries. The pathogenesis of PCOS has been linked to the effects of VDRs (TaqI, BsmI, FokI, ApaI and Cdx2 polymorphisms) on LH and SHBG levels, testosterone levels-,insulin resistance and serum insulin levels. Vitamin D deficiency increases PTH production, which is regulated through levels of serum calcium and vitamin $\mathrm{D}$. It has been suggested that the combination of vitamin D deficiency and dietary calcium insufficiency may be largely responsible for the menstrual abnormalities associated with PCOS. The low calcium intake might also contribute to the hormonal dysregulation that occurs in PCOS. VDRs play an important role in oestrogen production in the ovary. Vitamin D regulates oestrogen biosynthesis through direct regulation of the expression of the aromatase gene and by maintaining extracellular calcium homoeostasis In human ovarian tissue, 1,25-dihyroxyvitamin D3 stimulation of oestrogen and progesterone production and lack of effect on testosterone production may be explained by the augmentation of aromatase activity by vitamin D. Aromatase gene expression was decreased in PCOS follicles compared to controls, and they had increased levels of LH but decreased follicular production of progesterone and estradiol by preovulatory follicles, possibly because of the hyperluteinized microenvironment of PCOS follicles. As a result of these effects, vitamin D deficiency may exacerbate symptoms of PCOS.(8) 
While the exact mechanism underlying vitamin D and insulin resistance is not known, multiple cellular and molecular mechanisms have been proposed to explain the relationship. The biologically active form of vitamin $\mathrm{D}$, 1,25-dihydroxyvitamin D (1,25OHD) may enhance insulin action by enhancing insulin synthesis and release, increasing insulin receptor expression or suppression of proinflammatory cytokines that are believed to mediate insulin resistance. Vitamin D may also mediate insulin sensitivity by improving calcium status, increasing local production of $25 \mathrm{OHD}$, which leads to transcriptional regulation of specific genes or suppressing serum levels of PTH. a recent study there is substantial evidence supporting a relationship between vitamin D status and insulin sensitivity .

Vitamin D levels have also been negatively associated with insulin resistance (fasting insulin and HOMA-IR). A study by Hahn et al. ${ }^{[9]}$ also found that when grouping the women with PCOS according to 25OHD levels, lower levels of 25OHD were associated with insulin resistance and obesity. It has been suggested that obesity may have a confounding role in the relationship between 25OHD and insulin resistance in women with PCOS. However, one study showed that women with PCOS with severe vitamin D deficiency were more insulin resistant, independently of BMI and WHR and another showed that while 25OHD levels were lower in obese women with PCOS, in both the obese and non-obese women, 25OHD levels were negatively correlated with BMI and HOMA-IR, Wehr et al. also investigated this relationship using a multivariate regression analysis and found 25OHD levels were a significant and independent predictor for HOMA-IR along with BMI. This study suggests lower 25OHD levels are associated with higher insulin resistance. (8)

Insulin resistance is an essential feature of MS that has been linked to RA [38]. In a study that included 94 patients with RA, Dessein and Joffe [9] observed that insulin resistance was associated with markers for inflammation such as C- reactive protein and erythrocyte sedimentation rate and disease activity scores. On the other hand, betacell function showed an inverse correlation with DAS28 and swollen and painful joint. Chung et al. [10] studied insulin resistance in104 patients with RA and compared the results with those of 124 cases of systemic lupus erythematosus. They found that patients with RA have a higher insulin resistance index than systemic lupus erythematosus patients, and that insulin resistance was directly correlated with levels of interleukin 6, tumor necrosis factor-(TNF-) a, C-reactive protein, and erythrocyte sedimentation rate or coronary calcification Others studies have also confirmed the association between insulin resistance and RA

Vitamin D has immunologic activity independent of its crucial role in calcium regulation .Animal models of autoimmune disease have shown beneficial effects of vitamin $D$ as an immunosuppressant. For example, murine models of human RA demonstrated both decreased incidence and severity of disease in mice treated with active vitamin D . Evidence supporting an effect of vitamin D in RA specifically also is derived from clinical observations. Manolagas et al found that a significantly greater proportion of seropositive RA patients (76\%) had lymphocytes possessing vitamin D receptors compared with controls $(18 \%)$. Within the rheumatoid joint, the active form of vitamin $\mathrm{D}$ has been shown to be synthesized in RA synovium and is thought to be stimulated by interleukin-1 (IL-1) and/or IL-2 . Locally produced vitamin $\mathrm{D}$ may act in a paracrine manner to decrease $\mathrm{T}$ cell responsiveness through the inhibition of cellular proliferation and reduction in lymphokine production when confronted by an inappropriate and overly exuberant immune response

\section{Conclusion:-}

Vitamin D is not only a regulator of bone and mineral metabolism, but also a potent immunomodulator linked to many major human diseases including glucose homeostasis and insulin resistance. The evidence suggests the role of vitamin D in the pathogenesis of insulin resistance including several vitamin-D-related gene polymorphisms and vitamin-D-related metabolic and immune pathways. Supplementations of vitamin D may provide for suitable management to e insulin resistance. Insulin resistance encourages oxidative stress because hyperglycemia and higher levels of free fatty acids lead to ROS production. An increase in ROS generation resulting from hyperglycemia has been observed in women with PCOS [36], and insulin infusion in obese individuals has been shown to inhibit ROS production [37]. Thus, insulin may defend against pro-inflammatory responses to hyperglycemia by acting as an anti-inflammatory agent 


\section{References:-}

1. Dipanshu Sur* and Ratnabali Chakravorty, The Relationship between Vitamin D, Insulin Resistance and Infertility in PCOSWomen Gynecol Obstet (Sunnyvale)Volume 5 • Issue 5

2. Burghen GA, Givens JR, Kitabchi AE 1980 Correlation of hyperandrogenism with hyperinsulinism in polycystic ovarian disease. J Clin Endocrinol Metab 50:113-116

3. Michael L Traub Assessing and treating insulin resistance in women with polycystic ovarian syndrome World $\mathbf{J}$ Diabetes. 2011 Mar 15; 2(3): 33-40.

4. Evanthia Diamanti-Kandarakis and Andrea Dunaif

5. Insulin Resistance and the Polycystic Ovary Syndrome Revisited: An Update on Mechanisms and Implications; Endocrine Reviews, December 2012, 33(6):981-1030

6. Andrea Dunaif, Insulin Resistance and the Polycystic Ovary Syndrome: Mechanism and Implications for Pathogenesis* Endocr Rev (1997) 18 (6): 774-800

7. Velija-Asimi, Z. Evaluation of the association of vitamin D deficiency with gonadotropins and sex hormone in obese and non-obese women with polycystic ovary syndrome. Med. Glas. 2014, 11,170-176.

8. Tsakova, A.D.; Gateva, A.T.; Kamenov, Z.A. 25(OH) vitamin D levels in premenopausal women with polycystic ovary syndrome and/or obesity. Int. J. Vitam. Nutr. Res. 2012, 82, 399-404.

9. Rebecca L. Thomson; Simon Spedding; Jonathan D. Buckley .Vitamin D in the Aetiology and Management of Polycystic Ovary Syndrome Clin Endocrinol. 2012;77(3):343-350.

10. C. P. Chung, A. Oeser, J. F. Solus et al., "Inflammation- associated insulin resistance: differential effects in rheumatoid arthritis and systemic lupus erythematosus define potential mechanisms," Arthritis and Rheumatism, vol. 58, no. 7, pp. 2105-2112, 2008.

11. G. La Montagna, F. Cacciapuoti, R. Buono et al., "Insulin resistance is an independent risk factor for atherosclerosis in rheumatoid arthritis," Diabetes and Vascular Disease Research, vol. 4, no. 2, pp. 130-135, 2007.

12. Holick MF. Vitamin D: photobiology, metabolism, and clinical applications. In: DeGroot L, Besser H, Burger FIG, et al., eds. Endocrinology, $3{ }^{\text {rd }}$ ed; Philadelphia: WB Saunders, 1995: 900-1013.

13. Holick MF. Vitamin D deficiency. N Eng J Med 2007; 357: 266-281.

14. Holick MF. Sunlight and vitamin D for bone health and prevention of autoimmune diseases, cancers, and cardiovascular disease. Am J Clin Nutr 2004; 80 (6 suppl): 1678S-1688S.

15. Passeri G, et al. Low vitamin D status, high bone turnover, and bone fractures in centenarians. J Clin Endocrinol Metab 2003; 88: 5109-5115.

16. Dietary Supplement Fact Sheet: Vitamin D. Office of Dietary Supplements, National Institutes of Health, http://ods.od.nih.gov/factsheets/VitaminD-QuickFacts. Accessed September 2013.

17. Gerich JE: Hormónal control of homeostasis. IN Galloway JA, Potvin JH, Shuman CIR: Diabetes Mellitus, ninth edition. Eli Lilly Co, Indianapolis, 1988 pp 46-63

18. Rasmussen H, Zawalick KC. Ganesan S, Calle R, Zawalich WS: Physiology and pathophysiology of insulin secretion. Diab Care 13:655-666, 1990

19. Gammeltoft S: Insulin receptors: binding kinetics and structure-function relationship of insulin. Physiol Rev 64:1321-1378,1984

20. Rosen 0M: After insulin binds, Science 237:1452-1458, 1987

21. Schwartz MVV, Figlewicz D, Baskin DG, Woods SC, Porte D Jr. Insulin in the brain: A hormonal regulator of energy balance. Endocrin Rev 13:387-414,1992

22. Feldmann, M., Brennan, F.M., Maini, R.N. (1996). Rheumatoid arthritis. Cell 85, $307-310$.

23. Raptopoulou, A., Sidiropoulos, P., Katsouraki, M. (2007). Anti-citrulline antibodies in the diagnosis and prognosis of rheumatoid arthritis: evolving concepts.

24. Crit Rev Clin Lab Sci 44, 339 - 363.

25. Nishimura, K., Sugiyama, D., Kogata, Y., Tsuji, G., Nakazawa, T., Kawano, S., Saigo, K., Morinobu, A., Koshiba, M., Kuntz, K.M., Kamae, I., Kumagai, S. (2007). Meta-analysis: diagnostic accuracy of anti-cyclic citrullinated peptide antibody and rheumatoid factor for rheumatoid arthritis. Ann Intern Med 146, 797 - 808. 